\title{
Endoscopic ultrasound guided gastrojejunostomy
}

\author{
Enad Dawod ${ }^{1}$, Jose M. Nieto ${ }^{2}$ \\ ${ }^{1}$ Weill Cornell Medicine, Department of Gastroenterology and Hepatology, New York, NY, USA; ${ }^{2}$ Borland Groover Clinic, Advanced Therapeutic \\ Endoscopy Center, Jacksonville, FL, USA \\ Contributions: (I) Conception and design: All authors; (II) Administrative support: JM Nieto; (III) Provision of study materials or patients: JM Nieto; \\ (IV) Collection and assembly of data: E Dawod; (V) Data analysis and interpretation: All authors; (VI) Manuscript writing: All authors; (VII) Final \\ approval of manuscript: All authors. \\ Correspondence to: Jose M. Nieto, DO, FACP, FACG, AGAF, FASGE. Borland Groover Clinic, Advanced Therapeutic Endoscopy Center, \\ Jacksonville, FL, USA. Email: drjnieto@yahoo.com.
}

\begin{abstract}
Endoscopic ultrasound guided gastrojejunostomy (EUS-GJ) has been mostly employed in the treatment of benign and malignant gastric outlet obstruction (GOO). Additionally, EUS-GJ has been used in the treatment of afferent loop syndrome. EUS-GJ has been employed in the treatment of benign and malignant GOO. EUS-GJ has also been used in the treatment of afferent loop syndrome. EUS-GJ involves obtaining access to the jejunum endoscopically and sonographically from the stomach, using a biflanged lumen apposing metal stent (LAMS). This is achieved through a newly formed fistulous tract. Thus creating a gastrojejunal bypass fully endoscopically. Several techniques have been described in preforming EUS-GJ but no method has been shown to be superior. In afferent loop syndrome initial data show high technical and clinical success rates and decreased need for reintervention. With malignant GOO, data available from the limited number of studies conducted demonstrated he superiority of EUS-GJ when compared to standard enteral stenting in terms of decreasing recurrence of GOO and need for reintervention. With benign GOO, EUS-GE offers a solution to the long-term patency and recurrence issue encountered with enteral stenting and endoscopic balloon dilation. EUS-GJ offers a less invasive approach than surgery for the treatment of several etiologies, giving the patients the option to avoid adverse events associated with surgery.
\end{abstract}

Keywords: Endoscopic ultrasound (EUS); gastrojejunal anastomosis

Received: 06 September 2018; Accepted: 13 November 2018. Published: 21 November 2018.

doi: $10.21037 /$ tgh.2018.11.03

View this article at: http://dx.doi.org/10.21037/tgh.2018.11.03

Endoscopic ultrasound guided gastrojejunostomy or gastroenterostomy (EUS-GJ, EUS-GE) is novel endoscopic procedure that has recently emerged as a new treatment for some benign and malignant etiologies. For the most part, EUS-GJ has been employed in the treatment of benign and malignant gastric outlet obstruction (GOO) (1). Additionally, EUS-GJ has been used in the treatment of afferent loop syndrome (2).

EUS-GJ involves obtaining access to the jejunum endoscopically and sonographically from the stomach, using a biflanged lumen apposing metal stent (LAMS). This is achieved through a newly formed fistulous tract. Thus creating a gastrojejunal bypass fully endoscopically.

EUS-GJ has been validated in several animal studies and in some case series (3-7). Recently the multicenter studies that have been published mainly highlighting the use of EUS-GJ in treatment of GOO and afferent loop syndrome.

\section{Technique}

The development of the lumen-apposing self-expandable fully covered metal stent (AXIOS ${ }^{\mathrm{TM}}$, Boston Scientific Corp., Marlborough, MA, USA) has broadened the scope of what could be done endoscopically. The design of the stent allows adequate luminal apposition thus ensuring the creation of a fistulous tract between the two lumens. This is facilitated by the design of the stent, as there are two wide flanges on both sides, which provides adequate anchoring 

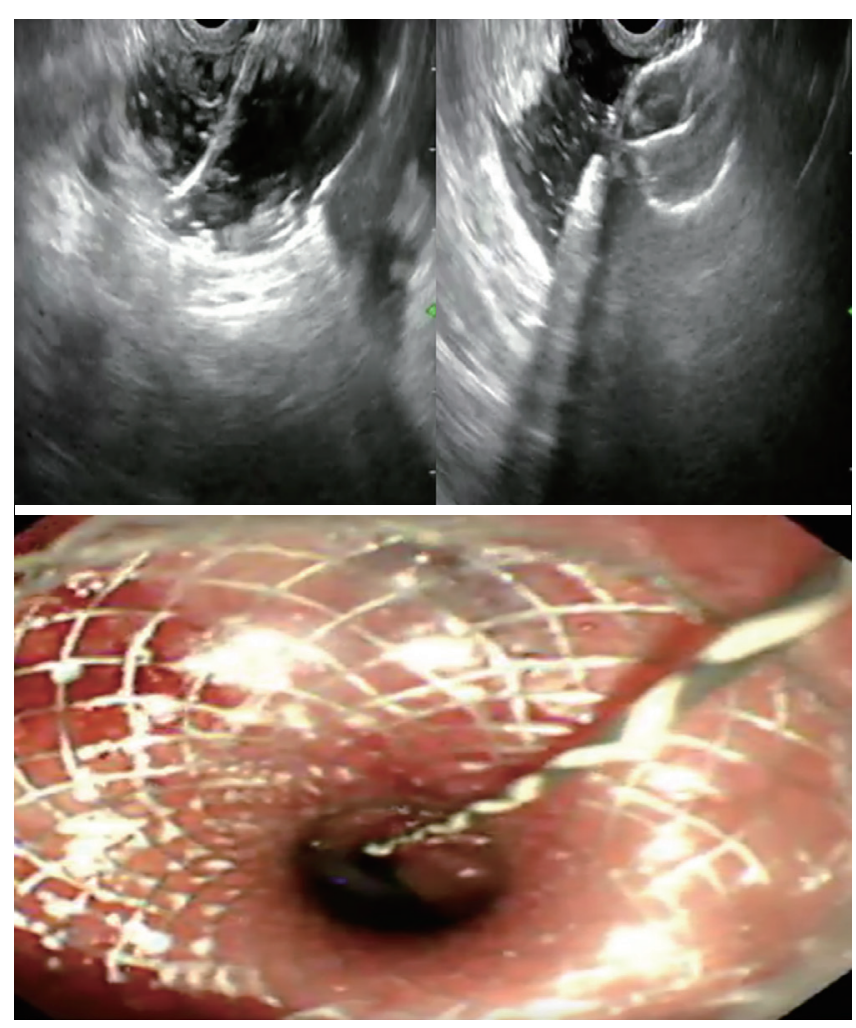

Figure 1 Steps of EUS-GJ. EUS-GJ, endoscopic ultrasound guided gastrojejunostomy.

between the two connected structures. The stent could be of 10 or 15 or $20 \mathrm{~mm}$ in size and when deployed through a French catheter the flanges expands into a larger diameter, which further secures the stent and prevents migration.

The targeted jejunal loop is identified using different approaches. All the techniques employed share in the common the use of a linear therapeutic endoscope and LAMS. The approach can be generally divided into direct assisted and the unassisted approach. The assisted approach involves intraluminal canalization of the stricture while the unassisted approach involves direct determination of the jejunal loop (3) (Figure 1).

The direct endoscopic ultrasound puncture technique involves advancing a linear endoscope into the stomach. The targeted jejunal loop is then identified endosonographically. A 19 gauge of the needle is then used to access the jejunum. A 22-gauge needle puncture could be used to distend jejunum before attempting again using the 19-gauge needle. Contrast injection is used to confirm the location before creating the fistulous tract using the LAMS (3).

The hybrid rendezvous approach uses an ultra thin scope. The ultra thin scope is advanced through a previously placed gastrostomy site or perorally into the stomach and then past the stricture into the jejunum in the case of GOO. Water is used to distend the lumen of the bowel. A 19 gauge of the needle is then used to access the jejunum after being identified endosonographically. The location within the jejunum is confirmed endoscopically. A guidewire was advanced through the needle and coiled within the bowel lumen. This is followed by the creation of a fistulous tract using the enhanced electrocautery LAMS delivery system (3).

Natural orifice transluminal endoscopic or free hand technique is another approach used to locate the jejunum. A 19-gauge needle under EUS guidance is used to create a transgastric puncture into the stomach. A guidewire is inserted into peritoneum and the linear echoendoscope is exchanged for a double-channel gastroscope over the wire. Through the second channel, a needle knife is inserted to make an incision on the gastric wall and the gastrostomy is dilated. Gastroscope is then passed into the peritoneum and used to locate and incise a jejunal loop with a needle knife. This is followed by subsequent advancement and coiling of guidewire in jejunum. The gastrojejunostomy is then created by deploying the distal flange of the LAMS in the jejunum followed by pulling back to the stomach where the proximal flange is deployed (8).

Balloon or nasobiliary drain catheter approach involves passing an endoscope into the stomach. A guidewire is coiled under fluoroscopic guidance into the jejunum past the stricture, under direct endoscopic if possible or with the fluoroscopic assistance if needed. A dilating balloon, biliary extraction balloon or nasobiliary drain catheter is then advanced over the wire into the jejunum under fluoroscopic guidance. Balloon catheters require removal of endoscope prior to insertion. However, with nasobiliary drain catheters are advanced through the endoscope, which are then removed afterwards. This is followed by advancing a linear echoendoscope into the stomach alongside the balloon or catheter. Contrast injection into the balloon or directly into the jejunal lumen through the nasobiliary drain is used to identify the jejunal loop echo sonographically and fluoroscopically. The loop is then accessed with a 19-gauge needle, and a second guidewire is advanced through it into the jejunal lumen for over-the-wire LAMS insertion If the cautery-tipped LAMS delivery system was is not available, the fistulous tract is serially dilated with a needle-knife and a balloon. Conversely, if the cautery-tipped LAMS 


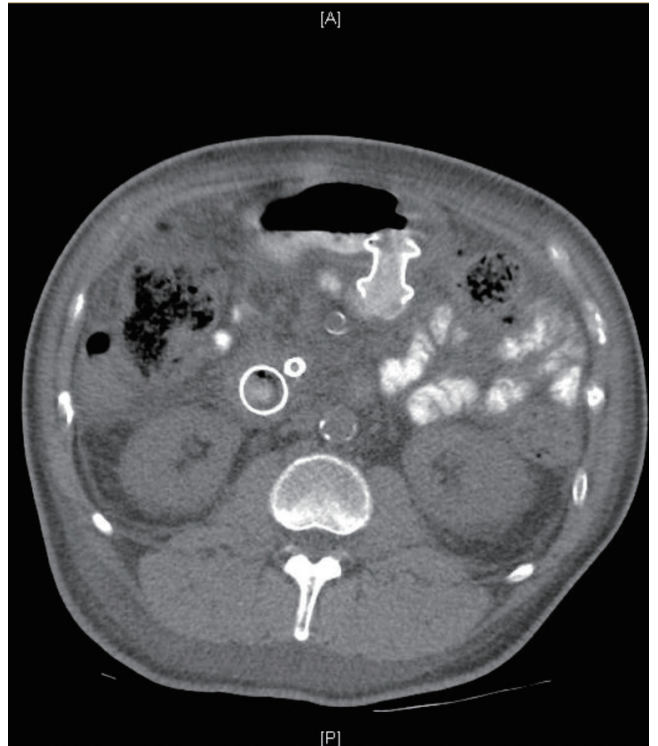

Figure 2 CT abdomen after EUS-GJ showing the LAMS. EUSGJ, endoscopic ultrasound guided gastrojejunostomy; LAMS, lumen apposing metal stent.

are available, the delivery system is inserted in one step without prior dilation, either over the wire or freehand, as previously described. A LAMS is deployed, and the balloon is dilated to its diameter (3) (Figure 2).

\section{GOO}

GOO can be due to benign or malignant causes. Benign causes include peptic ulcer disease, chronic pancreatitis, caustic injury and NSAID injury. Since the identification of Helicobacter Pylori and the increase of use of proton pump inhibitors, benign entities become the less common cause of GOO than malignant (9).

GOO has been conventionally treated using two methods, which are surgery and Endoscopic enteral stenting or balloon dilation. The surgical method is associated with a high complications rate. Endoscopic placement of an enteral self-expanding metal stent (SEMS) is associated with fewer complications, shorter hospitalization however there is a concern about long term patency and recurrence rates requiring reintervention. Endoscopic balloon dilation is another endoscopic modality that is effective in treating GOO. However the success of endoscopic balloon dilation varies with etiology as with GOO caused by caustic injury or GOO secondary to chronic pancreatitis (9).

Recently EUS-GJ has emerged as a new treatment
Table 1 Outcomes in patients who undergone EUS-GJ for the treatment of GOO

\begin{tabular}{lcccc}
\hline Name of author & $\begin{array}{c}\text { Number of } \\
\text { patients }\end{array}$ & $\begin{array}{c}\text { Clinical } \\
\text { success } \%\end{array}$ & $\begin{array}{c}\text { Technical } \\
\text { success \% }\end{array}$ & $\begin{array}{c}\text { Adverse } \\
\text { event \% }\end{array}$ \\
\hline Khashab et al. (10) & 10 & 90 & 90 & 0 \\
Itoi (11) & 20 & 90 & 90 & 2 \\
Tyberg et al. (3) & 26 & 85 & 92 & 11.5 \\
Chen et al. (12) & 30 & 83.3 & 86.7 & 10 \\
Khashab et al. (1) & 30 & 87 & 87 & 16 \\
Chen et al. (9) & 26 (benign) & 84 & 96.2 & 30 \\
\hline
\end{tabular}

modality for patients with GOO. Several case reports and more recently few retrospective studies demonstrated promising from the use of EUS-GJ in treatment benign and malignant GOO (Table 1). EUS-GE offers a less invasive approach than surgery without the associated morbidity. EUS-GE offers as solution to the long-term patency and recurrence issue encountered with enteral stenting and endoscopic balloon dilation.

Tyberg et al. published a multicenter international experience on 26 patients (17 with malignant obstruction) reported a $88 \%$ clinical success rate and technical success of $92 \%$. For 2 patients symptoms did not improve despite the patency of the GJ. Adverse event occurred in 3 patients (3). Itoi $e t a l$. were the first to publish a prospective study on a 20 patients who undergone EUS-GJ for the treatment of malignant GOO and reported a $90 \%$ clinical success and technical success rates (11). Before that a study was published on 10 patients ( 7 with benign GOO), they also reported a $90 \%$ clinical success and technical success rates with no adverse events reported (10).

A multicenter retrospective compared 30 who undergone EUS-GE and 52 who undergone enteral stenting for the treatment of GOO. ES and EUS-GE were comparable in technical success and clinical success however EUSGE was associated with less recurrence and need for reintervention (12).

Another international retrospective study compared EUS-GE to surgical gastrojejunostomy. 30 patients underwent EUS-GE while 63 undergone surgical gastrojejunostomy. Surgical gastrojejunostomy had higher technical success (100\% vs. $87 \%)$. The two methods were not statistically different in terms of clinical success, hospital stay length, recurrence rates and time for re intervention (1).

Most recently, an international retrospective series 
involving 5 tertiary centers looked at 26 patients who undergone EUS-GE for management of only benign GOO. Etiologies included strictures from chronic pancreatitis, surgical anastomosis, peptic ulcer disease acute pancreatitis, superior mesentery artery syndrome, caustic injury and hematoma. Clinical success was achieved in $84.0 \%$ of patients. $4.8 \%$ was the rate of unplanned reintervention and $3 \mathrm{AE}$ were reported (9).

In regards to benign GOO, where the success of endoscopic balloon dilation and enteral stenting varies with etiology, EUS-GJ could offer a solution to this issue given the fact that it bypasses the site of obstruction or stenosis which could be not be amenable to stent placement or dilation given severe chronic fibrosis as in the case with caustic injury, thus maintaining functional anastomosis away from the obstructed area.

Furthermore, in some cases multiple episodes of balloon dilation are required which means the patient would have to endure several procedures unlike EUS-GJ where less number of procedures is required. In patients who fail surgical treatment for GOO or do not qualify for it, there are limited endoscopic options. EUS-GJ is a promising modality for patients with GOO who failed balloon dilation or enteral stent placement (9).

In terms of malignant, data available from the limited number of studies conducted demonstrated he superiority of EUS-GJ when compared to standard enteral stenting in terms of decreasing recurrence of GOO and need for reintervention (12). EUS-GJ is a safe and minimally invasive modality that would be an ideal alternative for surgery in patients who fail standard endoscopic stenting. It provides long duration of luminal patency with no high risk of adverse events and without the morbidity associated with surgery. The longer duration of luminal patency might be due to the fact that LAMS is placed away from the site of obstruction which would be always susceptible to tumor ingrowth and tissue hyperplasia (3).

Some considerations need to be taken in mind when using EUS-GJ for treating GOO. The location of the targeted jejunely loop must be within $2 \mathrm{~cm}$ of the gastric wall by EUS, which is necessary in order to ensure complete apposition of the two lumens that are connected through the LAMS. Direct puncture through the tumor carries the risk of complications such as bleeding and incomplete apposition. The presence of downstream luminal obstruction distal to the jejunal puncture site may result in making the procedure ineffective in providing symptomatic relief. In the presence of severe outlet obstruction, the endoscopist will encounter difficulty in advancing a wire to the jejunum may render the procedure not feasible. Lastly if the area of obstruction is in the small bowel around the ligament of treitz which is adjacent to stomach, the procedure would be more challenging and sometimes not possible (8).

\section{Afferent loop syndrome}

Afferent loop syndrome usually occurs in patients who undergone surgical gastrojejunostomy or Roux-en-Y reconstruction. Afferent limb is usually part of the distal duodenum or proximal jejunum that is left behind the gastrojejunostomy. Afferent limb syndrome manifests as a pancreatobiliary symptoms. Afferent limb syndrome is usually caused by mechanical obstruction of the afferent limb or kinking. Complete obstruction occurs which leads to cholangitis, pancreatitis, perforation, and necrosis (2).

Afferent loop syndrome is conventionally treated surgically. Endoscopic modalities have also been in the rise, offering a less invasive approach to management. Endoscopic approaches included balloon dilation and stenting with double pigtail and/or a metal stent. The rise of LAMS have allowed for the use of EUS-GE in treating afferent loop syndrome.

The procedure can be preformed using cautery enhanced or non-cautery enhanced LAMS. Dilation of the of the tract is preformed using a balloon catheter or a cystatome over a guidewire which is followed by LAMS deployment. Regardless of the technique used the afferent limb is distended using a mixed solution of contrast saline and methylene blue (2).

The use of EUS-GJ in treatment of afferent limb syndrome has been described in few case reports and small case series $(2,13)$. Recently a multicenter international experience on 18 patients has been published. They reported a clinical success of $89 \%$ and $100 \%$ technical success. 3 adverse events were recorded. The same cohort of patients was compared to 17 patients enteroscopy-assisted luminal stenting (EALS). The EUS-GE group had more clinical success and less need for reintervention. There was no statistically significant difference in procedure times and technical success (2).

\section{Discussion}

EUS-GJ offers a less invasive approach than surgery, giving the patients the option to avoid the morbidity of having to 
undergo surgical bypass and the associated wound healing complications and other adverse events associated with surgery. In the terminally Ill patients with contraindications to surgery, EUS-GJ is new effective long lasting treatment modality. From an economic stand of point, EUS-GJ is less expensive than surgery.

EUS-GJ is a promising rising procedure. Initial data shows high technical and clinical success rates with low adverse events rate. EUS-GJ remains a highly technically challenging endoscopic procedure and is only to be preformed by highly experienced advanced endoscopists with experience in EUS and fluoroscopy at tertiary care centers in the setting of appropriate support. Given the presence of many techniques among different advanced endoscopists to preform EUS-GJ, there is no clarity on which method is superior in achieving clinical success and technical success and shorter procedural times. More studies need to be done as the technique is perfected. Adverse events reported from EUS-GJ included peritonitis, bleeding and surgery and death, stent misdeployment of stent into peritoneum, cholangitis, abdominal pain and fever $(1,3,9,12)$.

The choice of stent is of paramount importance and an appropriate lumen apposing metal stent must be used to ensure adequate apposition and maintain patency. Patient related factors must be taken in account in assessing whether to preform EUS-GJ such as in the case of GOO where severe outlet obstruction, presence of ascites or downstream obstruction will render the procedure not feasible or infective in causing clinical improvement. The data currently is mostly from retrospective studies. More prospective randomized trials need to be done to compare EUS-GJ to other modalities and to study long-term technical clinical success, complications, and safety of its removal.

And the need for reintervention before EUS-GJ could become an established standard of care. Until then, EUSGJ should be reserved for patients who fail or do not qualify for standard endoscopic treatment. In regards to GOO obstruction, most studies focused on malignant causes of GOO. More studies should focus on benign GOO, especially when we take into consideration the fact those different etiologies of benign GOO cause difference in outcomes.

\section{Acknowledgements}

None.

\section{Footnote}

Conflicts of Interest: JM Nieto, consultant for Boston Scientific. E Dawod has no conflicts of interest to declare.

\section{References}

1. Khashab MA, Bukhari M, Baron TH, et al. International multicenter comparative trial of endoscopic ultrasonography-guided gastroenterostomy versus surgical gastrojejunostomy for the treatment of malignant gastric outlet obstruction. Endosc Int Open 2017;5:E275-81.

2. Brewer Gutierrez OI, Irani SS, Ngamruengphong S, et al. Endoscopic ultrasound-guided entero-enterostomy for the treatment of afferent loop syndrome: a multicenter experience. Endoscopy 2018;50:891-5.

3. Tyberg A, Perez-Miranda M, Sanchez-Ocaña R, et al. Endoscopic ultrasound-guided gastrojejunostomy with a lumen-apposing metal stent: a multicenter, international experience. Endosc Int Open 2016;4:E276-81.

4. Binmoeller KF, Shah JN. Endoscopic ultrasoundguided gastroenterostomy using novel tools designed for transluminal therapy: a porcine study. Endoscopy 2012;44:499-503.

5. Itoi T, Ishii K, Tanaka R, et al. Current status and perspective of endoscopic ultrasonography-guided gastrojejunostomy: endoscopic ultrasonography-guided double-balloon-occluded gastrojejunostomy (with videos). J Hepatobiliary Pancreat Sci 2015;22:3-11.

6. Luo H, Pan Y, Min L, et al. Transgastric endoscopic gastroenterostomy using a partially covered occluder: a canine feasibility study. Endoscopy 2012;44:493-8.

7. Vanbiervliet G, Bonin EA, Garcès R, et al. Gastrojejunal anastomosis using a tissue-apposing stent: a safety and feasibility study in live pigs. Endoscopy 2014;46:871-7.

8. Amin S, Sethi A. Endoscopic Ultrasound-Guided Gastrojejunostomy. Gastrointest Endosc Clin N Am 2017;27:707-13.

9. Chen YI, James TW, Agarwal A, et al. EUS-guided gastroenterostomy in management of benign gastric outlet obstruction. Endosc Int Open 2018;6:E363-8.

10. Khashab MA, Kumbhari V, Grimm IS, et al. EUS-guided gastroenterostomy: the first U.S. clinical experience (with video). Gastrointest Endosc 2015;82:932-8.

11. Itoi $\mathrm{T}$, Ishii $\mathrm{K}$, Ikeuchi $\mathrm{N}$, et al. Prospective evaluation of endoscopic ultrasonography-guided double-balloonoccluded gastrojejunostomy bypass (EPASS) for malignant gastric outlet obstruction. Gut 2016;65:193-5. 
12. Chen YI, Itoi T, Baron TH, et al. EUS-guided gastroenterostomy is comparable to enteral stenting with fewer re-interventions in malignant gastric outlet obstruction. Surg Endosc 2017;31:2946-52.

13. Shah A, Khanna L, Sethi A. Treatment of afferent limb

doi: $10.21037 / \operatorname{tgh} .2018 .11 .03$

Cite this article as: Dawod E, Nieto JM. Endoscopic ultrasound guided gastrojejunostomy. Transl Gastroenterol Hepatol 2018;3:93. syndrome: novel approach with endoscopic ultrasoundguided creation of a gastrojejunostomy fistula and placement of lumen-apposing stent. Endoscopy 2015;47 Suppl 1 UCTN:E309-10. 